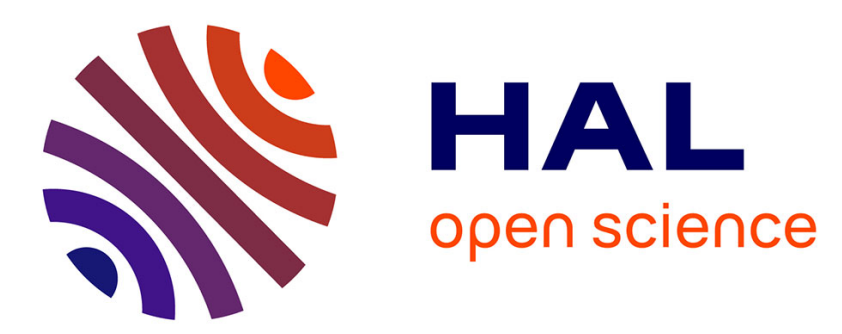

\title{
Derivation of third-order nonlinear susceptibility of thin metal films as a delayed optical response
}

\author{
Matteo Conforti, Giuseppe Della Valle
}

\section{To cite this version:}

Matteo Conforti, Giuseppe Della Valle. Derivation of third-order nonlinear susceptibility of thin metal films as a delayed optical response. Physical Review B: Condensed Matter and Materials Physics (1998-2015), 2012, 85 (24), 10.1103/PhysRevB.85.245423 . hal-02395032

\section{HAL Id: hal-02395032 \\ https://hal.science/hal-02395032}

Submitted on 5 Dec 2019

HAL is a multi-disciplinary open access archive for the deposit and dissemination of scientific research documents, whether they are published or not. The documents may come from teaching and research institutions in France or abroad, or from public or private research centers.
L'archive ouverte pluridisciplinaire HAL, est destinée au dépôt et à la diffusion de documents scientifiques de niveau recherche, publiés ou non, émanant des établissements d'enseignement et de recherche français ou étrangers, des laboratoires publics ou privés. 


\title{
Derivation of third-order nonlinear susceptibility of thin metal films as a delayed optical response
}

\author{
Matteo Conforti* \\ CNISM, Dipartimento di Ingegneria dell' Informazione, Università di Brescia, Via Branze 38, 25123 Brescia, Italy
}

Giuseppe Della Valle

Dipartimento di Fisica and IFN-CNR, Politecnico di Milano, Piazza L. da Vinci 32, I-20133 Milan, Italy

(Received 15 September 2011; revised manuscript received 22 February 2012; published 11 June 2012)

\begin{abstract}
Metals typically have very large optical nonlinearities, whose origin is mainly of thermal character. We model the cubic nonlinearity of thin metal films by means of a delayed response derived from an improved version of the classic two-temperature model. We validate our model by comparison with ultrafast pump-probe experiments on gold films.
\end{abstract}

DOI: 10.1103/PhysRevB.85.245423

Noble-metal nanostructures, such as thin films, gratings, multilayers, and nanoparticles, have been largely exploited in optics, especially within the fields of plasmonics and metamaterials. The linear optical response of these structures has been extensively studied both theoretically and experimentally, leading to the demonstration of unprecedented possibilities for extreme light concentration and manipulation (see Ref. 1 and references therein). Also, the huge nonlinear optical response of metallic nanostructures after intense excitation with fs-laser pulses has attracted increasing attention in view of the potential to achieve ultrafast all-optical control of light beams. ${ }^{2-5}$

Usually, the nonlinear response of the metal is modeled as a pure Kerr effect, ${ }^{2-4}$ where the nonlinear polarization is proportional to the cube of the electric field: $P_{N L}=\varepsilon_{0} \chi^{(3)} E^{3}$. In fact, this model, though perfectly describing nonresonant nonlinearities of electronic type that respond extremely fast (on the subfemtosecond time scale) to a driving electric field, turned out to be unsuitable for fs and ps optical pulses. Actually, the values of the $\chi^{(3)}$ coefficient, measured by the $\mathrm{z}$-scan technique, which can be found in the literature, differ by up to two orders of magnitude, ${ }^{3,6-8}$ clearly demonstrating that an instantaneous Kerr model is inadequate to describe the nonlinear response of metallic nanostructures.

Pump-probe experiments in thin films ${ }^{9-11}$ and nanoparticles $^{12,13}$ reveal that the nonlinearity of metals is due to the smearing of the electron distribution induced by intense optical absorption, resulting in a modulation of the interband and intraband transition probabilities with subsequent variation of the dielectric permittivity. The temporal dynamics of the system has been accurately interpreted according to the two-temperature model (TTM), which describes the energy balance between the electron bath and the lattice by means of two differential equations governing the temporal evolution of the electron and lattice temperatures through a coupling parameter related to the electron-phonon scattering rate. ${ }^{9,14}$ The TTM indicates that the nonlinear response is dominated by a delay mechanism, but a theoretical formulation in terms of a noninstantaneous $\chi^{(3)}$ susceptibility is still lacking.

In this paper we derive, from an improved version of the TTM, a delayed third-order nonlinear response suitable for the description of optically thin metallic structures. The outcome of our model is also quantitatively compared with experimental results from pump-probe spectroscopy on thin gold films.
Starting from Maxwell equations (written in mks units), neglecting transverse dimensions (i.e., considering the propagation of plane waves), we can obtain the $1 \mathrm{D}$ wave equation for the electric field $E(z, t)$ :

$$
\begin{aligned}
& \frac{\partial^{2} E(z, t)}{\partial z^{2}}-\frac{1}{c^{2}} \frac{\partial^{2}}{\partial t^{2}} \int_{-\infty}^{+\infty} E\left(z, t^{\prime}\right) \varepsilon\left(t-t^{\prime}\right) d t^{\prime} \\
& =\frac{1}{\varepsilon_{0} c^{2}} \frac{\partial^{2}}{\partial t^{2}} P_{N L}(z, t),
\end{aligned}
$$

where $c$ is the vacuum velocity of light, $\varepsilon_{0}$ is the vacuum dielectric permittivity, $\hat{\varepsilon}(\omega)=1+\hat{\chi}(\omega)$, and $\hat{\chi}(\omega)$ is the linear electric susceptibility (the hat standing for Fourier transform). In the perturbative regime, the nonlinear polarization can be expanded in Volterra series, accounting for small and noninstantaneous nonlinearity. ${ }^{15}$ Considering only third-order nonlinearity, nonresonant, incoherent (intensity-dependent) nonlinear effects can be included by assuming the following functional form for the third-order polarization:

$$
P_{N L}(z, t)=\varepsilon_{0}\left[\int_{-\infty}^{+\infty} \chi^{(3)}\left(t_{1}\right) E^{2}\left(t-t_{1}, z\right) d t_{1}\right] E(z, t) .
$$

With the aim of validating our derivation of the nonlinear response function by comparison with pump-probe experiments, we assume that the electric field is the sum of a powerful pump $A$ and a weak probe $B: E(z, t)=\frac{1}{2} A(z, t) e^{i \omega_{a} t}+$ $\frac{1}{2} B(z, t) e^{i \omega_{b} t}+$ c.c., with $|A| \gg|B|$. In the slowly varying envelope approximation $\left(\left|\partial_{t} A\right| \ll \omega_{a}|A|,\left|\partial_{t} B\right| \ll \omega_{b}|B|\right)$, the evolution equation for the probe becomes

$$
\frac{\partial^{2} B}{\partial z^{2}}+k_{b}^{2} B=-\frac{\omega_{b}^{2}}{2 c^{2}} \int_{-\infty}^{+\infty} \chi^{(3)}\left(t-t_{1}\right)\left|A\left(t_{1}, z\right)\right|^{2} d t_{1} B
$$

where $k_{b}^{2}=\omega_{b}^{2} \varepsilon_{b} / c^{2}=\omega_{b}^{2} \hat{\varepsilon}\left(\omega_{b}\right) / c^{2}$. In optically thin metallic structures we can assume that the pump is nondepleted, so that the nonlinear response is space-independent. By doing so, Eq. (3) can be written as

$$
\frac{\partial^{2} B}{\partial z^{2}}+\frac{\omega_{b}^{2}}{c^{2}}\left[\varepsilon_{b}+\Delta \varepsilon\left(|A(t)|^{2}\right)\right] B=0,
$$

where the time-dependent nonlinear dielectric constant change $\Delta \varepsilon$ is a convolution between the pump pulse intensity and the 
third-order nonlinear response of the system; that is,

$$
\Delta \varepsilon\left(|A(t)|^{2}\right)=\frac{1}{2} \int_{-\infty}^{+\infty} \chi^{(3)}\left(t-t_{1}\right)\left|A\left(t_{1}, z\right)\right|^{2} d t_{1} .
$$

Note that Eq. (4) is a linear wave equation for the probe $B$, where the time enters as a parameter.

In the following we demonstrate that the third-order nonlinear susceptibility can be derived from the extended two-temperature model, which describes the evolution of the electrons and lattice temperature of a metal after absorption of a laser pulse: ${ }^{9}$

$$
\begin{aligned}
C_{e} \frac{\partial T_{e}}{\partial t} & =-g\left(T_{e}-T_{l}\right)+a N, \\
C_{l} \frac{\partial T_{l}}{\partial t} & =g\left(T_{e}-T_{l}\right)+b N, \\
\frac{\partial N}{\partial t} & =-a N-b N+P(z, t),
\end{aligned}
$$

where $C_{e}$ and $C_{l}$ are the electronic and lattice heat capacities, $T_{l}$ is the lattice temperature, $g$ is the electron-phonon coupling constant, $N$ stands for the energy density stored in the nonthermalized part of the electronic distribution, $a$ is the electron gas heating rate, and $b$ is the electronphonon coupling rate. It is worth noting that separating the electronic distribution in a thermalized part (characterized by $T_{e}$ ) and a nonthermalized part $(N)$ may appear as a strong hypothesis. However, rigorous calculations based on the Boltzmann equation ${ }^{9,14}$ show the validity of this procedure for the temperature and energy ranges we are dealing with. $P$ is the absorbed energy density and is related to field intensity $I$ through $P(z, t)=(1-R-T) \alpha e^{-\alpha z} I(t)$; for a thin film, we can neglect the spatial dependence and assume a mean absorbed energy density $P(t)=(1-R-T) I(t) / d(d, R$, $T$, are the film thickness, reflection, and transmission). The energy density $N$ can be calculated as the convolution between the pump energy density and the thermalization response function $h_{t h}(t)=\exp \left[-t / \tau_{t h}\right] H(t)[H(t)$ being the Heaviside function], with $\tau_{t h}=1 /(a+b)$. The values $a, b$ can be derived from a more sophisticated extension of TTM proposed by Carpene. ${ }^{14}$ It is worth noting that the two approaches give rather similar results, but the rate equation approach ${ }^{9}$ is simpler. We obtain $a=1 / 2 \tau_{1}, \tau_{1}=E_{F}^{2} \tau_{0} /\left(\hbar \omega_{a}\right)^{2}$, and $b=$ $1 / \tau_{e p}$, where $E_{F}$ is the Fermi energy, $\tau_{0}=128 \sqrt{3} \pi^{2} \omega_{p}\left(\omega_{p}\right.$ the plasma frequency), and $\tau_{e p}$ is the electron-phonon energy relaxation time. The value of $\tau_{0}$ has been calculated using the Lindhard dielectric function in the framework of Fermi liquid theory under the random phase approximation. ${ }^{16}$ This value turns out to be underestimated, mainly because of the $d$-band screening; a more correct value can be extracted from experimental data. ${ }^{16}$ If $\Delta T_{e}=T_{e}-T_{a} \ll T_{a}$, it is possible to consider the thermal capacity of electrons $C_{e}=\gamma T_{e}=\gamma T_{0}$ nearly constant: In this way the system (6) is linear and can be solved exactly. $T_{0}$ can be estimated as a mean value between the minimum electronic temperature $T_{a}$ and the maximum value, which can be estimated by $T_{e, \max } \approx \sqrt{T_{a}^{2}+2 / \gamma \int P(t) d t}$. For most metals, including $\mathrm{Au}$ and $\mathrm{Ag}, C_{l} \gg C_{e}$, allowing us to write a simple expression for $\Delta T_{e}$ :

$$
\Delta T_{e}=\int N\left(t-t_{1}\right) \frac{C_{e}+a \tau_{t h} C_{l} e^{-t_{1} / \tau}}{C_{e}\left(C_{e}+C_{l}\right) \tau_{t h}} H\left(t_{1}\right) d t_{1},
$$

i.e., the convolution between the energy density $N$ and the twotemperature system response $h(t)=\frac{C_{e}+a \tau_{t h} C_{l} e^{-t / \tau}}{C_{e}\left(C_{e}+C_{l}\right) \tau_{t h}} H(t)$, being $\tau=g^{-1} C_{e} C_{l} /\left(C_{e}+C_{l}\right)$.

It is now possible to calculate the total response of the extended two-temperature system as the convolution of $h_{t h}$ and $h$ :

$$
\begin{aligned}
h_{t o t}(t)= & \frac{1}{C_{e}+C_{l}} \times\left[1-e^{-t / \tau_{t h}}\right. \\
& \left.+\frac{a C_{l} \tau \tau_{t h}}{C_{e}\left(\tau-\tau_{t h}\right)}\left(e^{-t / \tau}-e^{-t / \tau_{t h}}\right)\right] H(t) .
\end{aligned}
$$

This way the electronic temperature change is simply calculated as the convolution between the pump and the total response:

$$
\Delta T_{e}=\int P\left(t-t_{1}\right) h_{t o t}\left(t_{1}\right) d t_{1} .
$$

Assuming a Gaussian pump $P(t)=P_{0} \exp \left[-2\left(t / \tau_{p}\right)^{2}\right]$ we obtain

$$
\begin{aligned}
\Delta T_{e}= & \sqrt{\frac{\pi}{2}} \frac{P_{0} \tau_{p} / 2}{C_{e}+C_{l}}\left\{1+\operatorname{Erf}\left(\frac{\sqrt{2} t}{\tau_{p}}\right)\right. \\
& +a \frac{C_{l}}{C_{e}} \frac{\tau \tau_{t h}}{\tau-\tau_{t h}}\left[e^{\frac{\tau_{p}^{2}}{8 \tau^{2}}-\frac{t}{\tau}} \operatorname{Erfc}\left(\frac{\sqrt{2}}{\tau_{p}}\left(\frac{\tau_{p}^{2}}{4 \tau}-t\right)\right)\right. \\
& \left.\left.-e^{\frac{\tau_{p}^{2}}{8 \tau_{t h}^{2}}-\frac{t}{\tau_{t h}}} \operatorname{Erfc}\left(\frac{\sqrt{2}}{\tau_{p}}\left(\frac{\tau_{p}^{2}}{4 \tau_{t h}}-t\right)\right)\right]\right\}
\end{aligned}
$$

Following the same procedure a similar expression can be obtained also for the lattice temperature variation $\Delta T_{L}$ and the nonthermalized electron energy density $N$.

We tested the validity of our approach by studying the temperature changes of a thin gold film $(d=20 \mathrm{~nm})$ deposited on a sapphire substrate $\left(n_{s}=1.75\right)$, after irradiation with a pump pulse of $\lambda_{a}=950 \mathrm{~nm}$ wavelength, $\tau_{p}=140 \mathrm{fs}$ duration, and $F=30 \mu \mathrm{J} / \mathrm{cm}^{2}$ fluence. Parameters for the TTM were taken from the literature ${ }^{11,14}$ as $T_{a}=300 \mathrm{~K}$, $\gamma=70 \mathrm{~J} \mathrm{~m}^{-3} \mathrm{~K}^{-2}, C_{l}=2.5 \times 10^{6} \mathrm{~J} \mathrm{~m}^{-3} \mathrm{~K}^{-1}, g=2 \times$ $10^{16} \mathrm{~W} \mathrm{~m}^{-3} \mathrm{~K}^{-1}, \tau_{e p}=1.4 \mathrm{ps}, \tau_{0}=6.5 \mathrm{fs},{ }^{16} E_{F}=7.3 \mathrm{eV}$. It should be noted that material parameters are temperature dependent. ${ }^{17}$ However a consistent formulation of nonlinear phenomena within a perturbation theory implies considering a limited range of induced temperature variations, of the order of few tens of degrees. This allows us to approximate all temperature-dependent parameters as constants with good accuracy. Figure 1 shows the results of numerical solution of Eqs. (6), of numerical solution of the Carpene model, ${ }^{14}$ and of analytical formula (10). The agreement between the analytical approximation and TTM is remarkable, indicating that in this range of electronic temperature the assumption of a constant heat capacity is reasonable. The Carpene model and TTM give practically the same outcome for what concerns lattice temperature, but a small discrepancy can be noticed for what concerns the peak of the electronic temperature. This feature is mainly due to the quite long thermalization time $\tau_{t h}$ and will produce a slightly higher estimation of the maximum dielectric constant changes. In this figure we also show the time evolution of the energy density stored in the nonthermalized electrons $N$. It acts like a delayed effective pump for electron and lattice, 


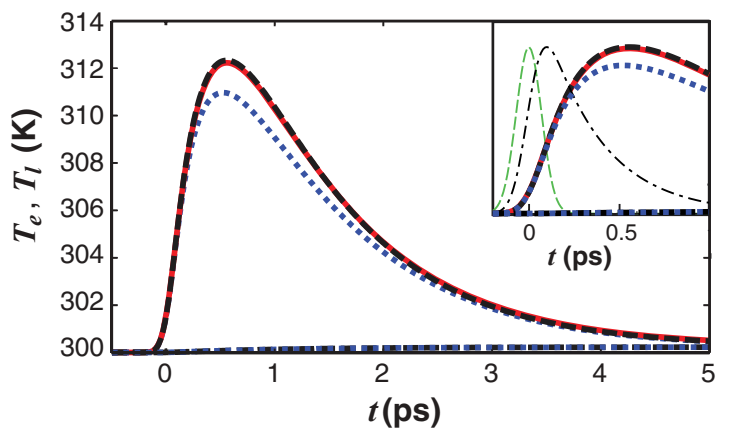

FIG. 1. (Color online) Temperature dynamics after irradiation of a thin gold film deposited on a sapphire substrate, from the TTM of Eq. (6) (dashed black curves), from the Carpene model (Ref. 14) (dotted blue curves), and from the analytical formula of Eq. (10) (solid red curve). Inset: Zoom-in of the initial temperature dynamics; normalized energy density stored in nonthermalized electrons (dashdot black curve); pump intensity profile (thin dashed green curve). See text for parameters.

slowing the rise of $\Delta T_{e}$ and $\Delta T_{l}$. The dynamics of $N$ is faster than the thermalized one and it is responsible for the small and quick dielectric permittivity changes observed when probing in a frequency range far from interband transitions. ${ }^{9}$

Variation in electronic temperature induces variation in the dielectric constant through interband (bound electrons) transitions. ${ }^{9}$ Additional contributions to the change in the dielectric constant are given by intraband transitions (which depend on lattice temperature) and by complex dynamics of nonthermalized electrons. ${ }^{18}$ These additional contributions are much smaller than the former and can be resolved only in spectral regions where interband transitions are not efficient. ${ }^{9}$ In order to obtain a reasonably simple model that however correctly grasps all the relevant phenomena, from now on we concentrate on interband transitions of thermalized electrons.

Energy injection from intra-band pump absorption smears the electron distribution around $E_{F}$, causing a reduction (increase) of the occupation probability for electron states at energy below (above) $E_{F}$. As a consequence, a modulation of interband transition probability is induced, with increased (decreased) absorption for transitions involving final states below (above) $E_{F}$. Interband transitions in noble metals are dominated by $d$-band to conduction band transitions near $L$ and $X$ points in the irreducible zone of the Brillouin cell. In the constant matrix element approximation, the variation of the imaginary part of the interband dielectric function of gold can be computed as follows: ${ }^{19}$

$$
\begin{aligned}
\Delta \varepsilon_{2}\left(\omega_{b}, T_{e}\right)= & \frac{4 \pi^{2} e^{2}}{3 m^{2} \omega_{b}^{2}}\left[\left|P_{L}\right|^{2} \Delta J_{L}\left(\omega_{b}, T_{e}\right)\right. \\
& \left.+\left|P_{X}\right|^{2} \Delta J_{X}\left(\omega_{b}, T_{e}\right)\right],
\end{aligned}
$$

where $m$ is the free electron mass, $P_{L}$ and $P_{X}$ are the electricdipole matrix elements, and $\Delta J_{L}$ and $\Delta J_{X}$ are the temperatureinduced variations of the joint density of states (JDOS) for $d$ band to conduction band transitions near $L$ and $X$, respectively.

Such variations can be computed as ${ }^{19}$

$$
\Delta J_{L, X}\left(\omega_{b}, T_{e}\right)=\int_{E_{L, X}^{\prime}}^{E_{L, X}^{\prime \prime}} D_{L, X}\left(E, \omega_{b}\right) \Delta P\left(E, T_{e}\right) d E,
$$

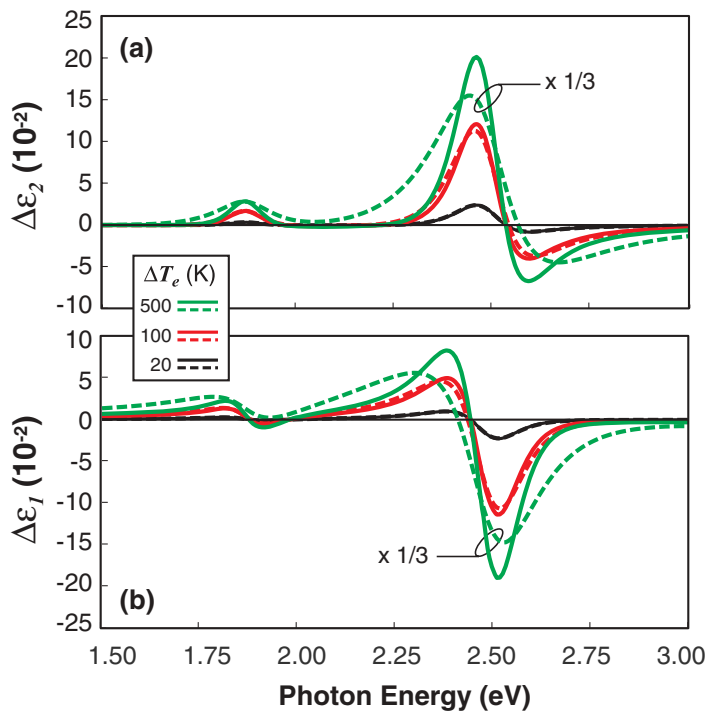

FIG. 2. (Color online) Spectral variation of the (a) imaginary part and (b) real part of the interband dielectric function of gold for three different values of $\Delta T_{e}$, computed from Eqs. (11)-(13). Linearized temperature dependence provided by Eq. (13) (solid lines) is compared with exact solution (dashed lines).

where $D_{L, X}\left(E, \omega_{b}\right)$ is the energy distribution of the joint density of states (EDJDOS) of the considered transitions (with respect to the energy of final state $E$ ), and $\Delta P\left(E, T_{e}\right)=f\left(E, T_{a}\right)-P\left(E, T_{e}\right)$ is the temperature-induced variation of the occupation probability for the final state [being $f\left(E, T_{e}\right)$ the Fermi-Dirac function], which for small temperature changes $\Delta T_{e}$ is approximated as $\Delta P\left(E, T_{e}\right) \simeq$ $-\left[\partial f\left(E, T_{e}\right) / \partial T_{e}\right]_{T_{e}=T_{a}} \Delta T_{e}$. This way, Eq. (11) can be factorized as

$$
\Delta \varepsilon_{2}\left(\omega_{b}, T_{e}\right)=\left[\frac{\partial \Delta \varepsilon_{2}\left(\omega_{b}, T_{e}\right)}{\partial T_{e}}\right]_{T_{e}=T_{a}} \Delta T_{e} .
$$

The EDJDOS was numerically computed under parabolic band approximation following the approach described in Ref. 19, taking effective masses, energy gaps, dipole matrix elements, and integration limits $E_{L, X}^{\prime}$ and $E_{L, X}^{\prime \prime}$ as reported in Ref. 20. The $\Delta \varepsilon_{2}$ resulting from Eqs. (11) or (13) as a function of photon energy for three different temperature variations is shown in Fig. 2(a). The variation of the real part of the interband dielectric function $\Delta \varepsilon_{1}$ computed by Kramers-Kronig analysis of $\Delta \varepsilon_{2}$ is reported in Fig. 2(b). Note that the approximation provided by Eq. (13) is accurate for $\Delta T_{e}$ as high as $100 \mathrm{~K}$.

For a quantitative validation of our model, we computed the transient differential reflection (transmission) $\Delta R / R(\Delta T / T)$ of the 20-nm thin gold film according to standard thin film formulas ${ }^{21}$ and Eqs. (10)-(13). The results are reported in Fig. 3, compared with experimental data taken from the literature ${ }^{9}$ and full-numerical computation from the extended TTM. The probe photon energy is chosen at the maximum response of the thin film; ${ }^{9}$ i.e., $\hbar \omega_{b}=2.48 \mathrm{eV}$ for $\Delta R / R$ and $\hbar \omega_{b}=2.43 \mathrm{eV}$ for $\Delta T / T$. We found that the analytical model is in quantitative agreement with experimental data, with only slight deviations in the long-time range $(t>4 \mathrm{ps})$ where the contribution from the lattice is dominant. 
By comparing Eqs. (13) and (5) taking into account Eq. (9) the third-order nonlinear susceptibility can be finally written as follows:

$$
\chi^{(3)}(t)=\frac{\partial \Delta \varepsilon}{\partial T_{e}} \alpha \operatorname{cn} \varepsilon_{0} h_{\mathrm{tot}}(t) .
$$

With Eq. (14) at hand, we can discuss the strength of the optical nonlinearity of gold predicted by our model. To compare with Kerr nonlinearity of standard materials, we consider continuous-wave pump and probe. In this case the nonlinear response is much faster than the fields, and the convolution reduces to the integral of $\chi^{(3)}(t)$. Assuming $C_{l} \gg C_{e}$ we obtain

$$
\hat{\chi}^{(3)}\left(\omega_{b} ; \omega_{a},-\omega_{a}, \omega_{b}\right)=\frac{\partial \Delta \varepsilon\left(\omega_{b}\right)}{\partial T_{e}} \alpha\left(\omega_{a}\right) c n\left(\omega_{a}\right) \varepsilon_{0} \frac{a \tau_{t h} \tau}{C_{e}}
$$

(the hat is added because assuming continuous-wave fields is equivalent to giving a frequency domain description of $\chi^{(3)}$ ). It should be stressed that this kind of nonlinearity is strongly dispersive, and can be assumed constant only for reasonably band-limited pulses centered around $\omega_{a}$ and $\omega_{b}$. Changing either pump or probe frequency results in a drastic change of $\hat{\chi}^{(3)}\left(\omega_{b}\right)$, confirming that this kind of third-order nonlinearity is not of Kerr type. To give an example of the values that $\hat{\chi}^{(3)}$ can assume, we consider an infrared pump $\lambda_{a}=950 \mathrm{~nm}$ and visible probe $\lambda_{b}=500 \mathrm{~nm}$, to obtain $\hat{\chi}^{(3)}\left(\omega_{b} ; \omega_{a},-\omega_{a}, \omega_{b}\right) \approx$ $(-8.4+11 i) \times 10^{-8}$ esu $=(-1.2+1.5 i) \times 10^{-15} \mathrm{~m}^{2} / \mathrm{V}^{2}$. This huge value (six orders of magnitude greater than in fused silica) is due to the resonance of the probe with interband transitions of gold. It is worth noting that for small frequency detuning from interband transitions $\chi^{(3)}$ can change its sign (see Fig. 2), whereas for large detuning its magnitude decreases drastically.

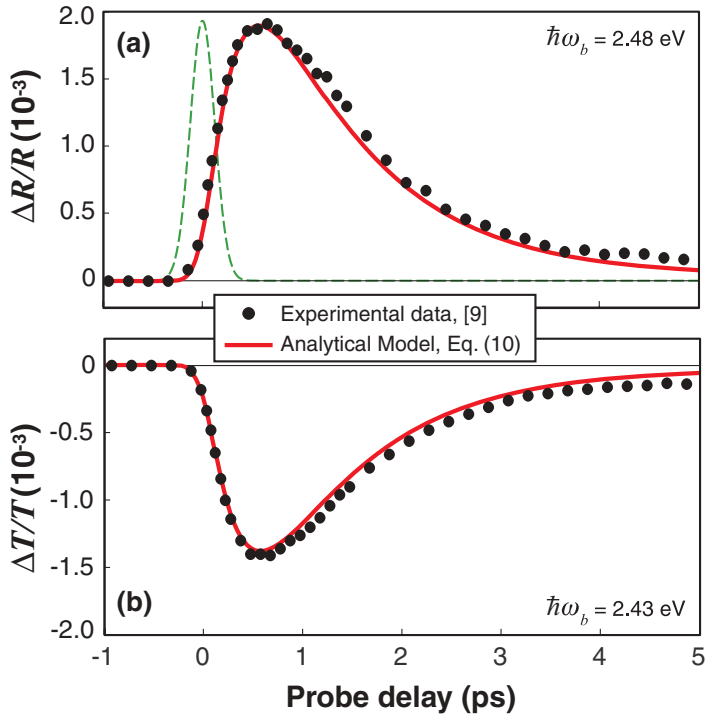

FIG. 3. (Color online) Transient (a) reflectivity and (b) transmissivity vs probe time delay, accounting for pump-probe crosscorrelation (shown as dashed green line).

To conclude, we introduced a theoretical model for the delayed nonlinear response in optically thin noble-metal structures. A noninstantaneous $\chi^{(3)}$ coefficient is derived from an extended version of the TTM and semiclassical theory of optical transitions in solids. Our theoretical predictions turned out to be in quantitative agreement with experimental results from pump-probe experiments in thin gold films.

This work was supported by Fondazione Cariplo through the projects "Engineering of Optical Nonlinearities in Plasmonic Metamaterials" (Grant No. 2010-0595) and "New Frontiers in Plasmonic Nanosensing" (Grant No. 2011-0338). *matteo.conforti@ing.unibs.it

${ }^{1}$ J. A. Schuller, E. S. Barnard, W. Cai, Y. C. Jun, J. S. White, and M. L. Brongersma, Nat. Mater. 9, 193 (2010).

${ }^{2}$ R. S. Bennik, Y.-K. Yoon, R. W. Boyd, and J. E. Sipe, Opt. Lett. 15, 1416 (1999).

${ }^{3}$ G. Yang, D. Guan, W. Wang, W. Wu, and Z. Chen, Opt. Mater. 25, 439 (2004).

${ }^{4}$ A. Husakou and J. Herrmann, Phys. Rev. Lett. 99, 127402 (2007).

${ }^{5}$ K. F. MacDonald, Z. L. Samson, M. I. Stockman, and N. I. Zheludev, Nat. Photon. 3, 55 (2009).

${ }^{6}$ T. K. Lee, A. D. Bristow, J. Hubner, and H. M. van Driel, J. Opt. Soc. B 23, 2142 (2006).

${ }^{7}$ D. D. Smith et al., J. Appl. Phys. 86, 6200 (1999).

${ }^{8}$ N. Rotenberg, A. D. Bristow, M. Pfeiffer, M. Betz, and H. M. van Driel, Phys. Rev. B 75, 155426 (2007).

${ }^{9}$ C.-K. Sun, F. Vallée, L. H. Acioli, E. P. Ippen, and J. G. Fujimoto, Phys. Rev. B 50, 15337 (1994).

${ }^{10}$ R. H. M. Groeneveld, R. Sprik, and A. Lagendijk, Phys. Rev. B 51, 11433 (1995).
${ }^{11}$ J. Hohlfeld, S.-S. Wellershoffm, J. Gudde, U. Conrad, V. Janke, and E. Matthias, Chem. Phys. 251, 237 (2000).

${ }^{12}$ N. Del Fatti, C. Voisin, M. Achermann, S. Tzortzakis, D. Christofilos, and F. Vallee, Phys. Rev. B 61, 16956 (2000).

${ }^{13}$ H. Baida, D. Mongin, D. Christofilos, G. Bachelier, A. Crut, P. Maioli, N. Del Fatti, and F. Vallee, Phys. Rev. Lett. 107, 057402 (2011).

${ }^{14}$ E. Carpene, Phys. Rev. B 74, 024301 (2006).

${ }^{15}$ R. W. Boyd, Nonlinear Optics, 2nd ed. (Academic, New York, 2003).

${ }^{16}$ W. S. Fann, R. Storz, H. W. K. Tom, and J. Bokor, Phys. Rev. B 46, 13592 (1992).

${ }^{17}$ Z. Lin, L. V. Zhigilei, and V. Celli, Phys. Rev. B 77, 075133 (2008).

${ }^{18}$ B. Rethfeld, A. Kaiser, M. Vicanek, and G. Simon, Phys. Rev. B 65, 214303 (2002).

${ }^{19}$ R. Rosei, Phys. Rev. B 10, 474 (1974).

${ }^{20} \mathrm{M}$. Guerrisi, R. Rosei, and P. Winsemius, Phys. Rev. B 12, 557 (1975).

${ }^{21} \mathrm{~F}$. Abeles, in Advanced Optical Techniques, edited by Van Heel (North-Holland, Amsterdam, 1967), Chap. 5, p. 144. 